\title{
Measurements of the Optical Performance of Prototype TES Bolometers for SAFARI
}

\author{
M.D. Audley • G. de Lange • M. Ranjan • J.-R. Gao • P. Khosropanah • \\ M.L. Ridder · P.D. Mauskopf • D. Morozov • S. Doherty • N. Trappe • \\ S. Withington
}

Received: 24 July 2013 / Accepted: 18 November 2013 / Published online: 26 November 2013

(C) Springer Science+Business Media New York 2013

\begin{abstract}
We have measured the optical response of prototype detectors for SAFARI, the far-infrared imaging spectrometer for the SPICA satellite. SAFARI's three bolometer arrays, coupled with a Fourier transform spectrometer, will provide images of a $2^{\prime} \times 2^{\prime}$ field of view with spectral information over the wavelength range 34-210 $\mu \mathrm{m}$. Each horn-coupled bolometer consists of a transition edge sensor (TES), with a transition temperature close to $100 \mathrm{mK}$, and a thin-film Ta absorber on a thermally-isolated silicon nitride membrane. SAFARI requires extremely sensitive detectors $\left(N E P \sim 2 \times 10^{-19} \mathrm{~W} / \sqrt{\mathrm{Hz}}\right)$, with correspondingly low saturation powers $(\sim 5 \mathrm{fW})$, to take advantage of SPICA's cooled optics. To meet the challenge of testing such sensitive detectors we have constructed an ultra-low background test facility
\end{abstract}

M.D. Audley $(\bowtie) \cdot$ G. de Lange $\cdot$ M. Ranjan

SRON Netherlands Institute for Space Research, Landleven 12, 9747 AD Groningen,

The Netherlands

e-mail: damian@sron.nl

J.-R. Gao · P. Khosropanah · M.L. Ridder

SRON Netherlands Institute for Space Research, Sorbonnelaan 2, 3584 CA Utrecht, The Netherlands

J.-R. Gao

Delft University of Technology, Lorentzweg 1, 2628 CJ Delft, The Netherlands

P.D. Mauskopf · D. Morozov

University of Cardiff, Cardiff CF24 3YB, UK

P.D. Mauskopf

Arizona State University, Tempe, AZ 85287, USA

S. Doherty · N. Trappe

National University of Ireland, Maynooth Co., Kildare, Ireland

S. Withington

Cavendish Laboratory, University of Cambridge, JJ Thomson Avenue, Cambridge, UK 
based on a cryogen-free high-capacity dilution refrigerator, paying careful attention to stray-light exclusion, shielding, and vibration isolation. For optical measurements the system contains internal cold $(3-30 \mathrm{~K})$ and hot $(\sim 300 \mathrm{~K})$ black-body calibration sources, as well as a light pipe for external illumination. We discuss our measurements of high optical efficiency in prototype SAFARI detectors and describe recent improvements to the test facility that will enable us to test the full SAFARI focalplane arrays.

Keywords Low-temperature detectors · Infra-red bolometers · Transition edge sensors $\cdot$ Optical testing

\section{Introduction}

The Japanese Space Agency's satellite observatory SPICA will use a large (3.5-m diameter) primary mirror cooled to $<6 \mathrm{~K}$ to enable high angular-resolution, skybackground limited observations of the cold dusty Universe in the mid- and farinfrared [1]. The SAFARI [2] instrument is a far-infrared imaging Fourier transform spectrometer (FTS) for the SPICA satellite with three detector arrays covering the wavelength ranges 34-60 (short wave), 60-110 (medium wave), and 110-210 $\mu \mathrm{m}$ (long wave). The detectors are Transition Edge Sensor (TES) bolometers [3]. To take advantage of SPICA's low-background cold mirror, SAFARI's short-wave detectors require a dark noise equivalent power $(N E P)$ less than $6.5 \times 10^{-19} \mathrm{~W} / \sqrt{\mathrm{Hz}}$, with a goal $N E P$ of $\sim 2 \times 10^{-19} \mathrm{~W} / \sqrt{\mathrm{Hz}}$ and correspondingly low saturation powers $(\sim 5 \mathrm{fW})$. We have measured the optical response of prototype detectors designed for SAFARI's short-wave band (33-60 $\mu \mathrm{m})$ array as shown in Fig. 1. A conical feedhorn and hemispherical backshort are used to couple radiation to the detector. These detectors have moderate sensitivity $(N E P=1-2 \mathrm{~W} / \sqrt{\mathrm{Hz}})$ and although they have been far surpassed in sensitivity by the latest detectors for SAFARI [4], they absorb radiation in the same way, and are thus representative for optical characterization.
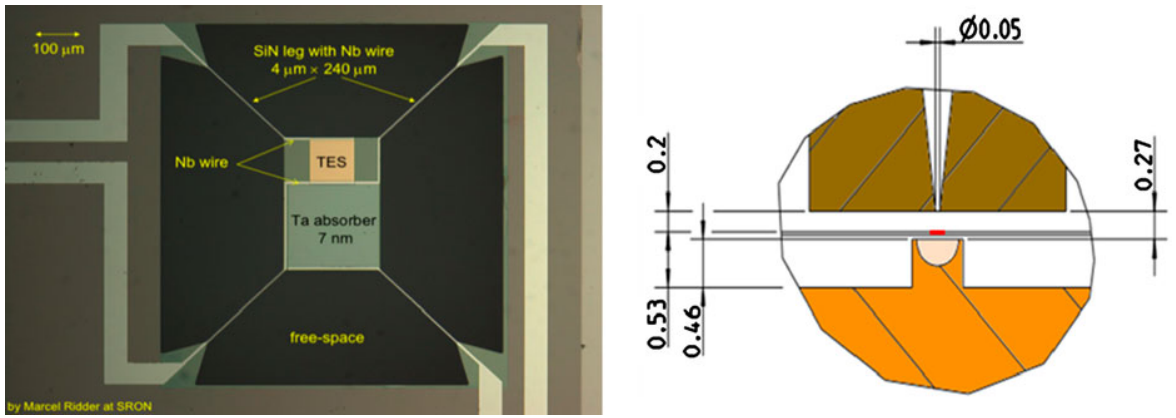

Fig. 1 Left: Photograph of a TES detector similar to the ones measured here. The Ta absorber is $200 \mu \mathrm{m}$ square. Right: Cross-section of feedhorn and spherical backshort showing position of detector with dimensions in $\mathrm{mm}$. The diameter of the spherical backshort is $0.5 \mathrm{~mm}$ and the horn is $4.5 \mathrm{~mm}$ long with an entrance aperture diameter of $0.45 \mathrm{~mm}$ (Color figure online) 


\section{Optical Test Setup}

The SAFARI Detector Test Facility is based on a Leiden Cryogenics dilution refrigerator with a cooling power of $\sim 200 \mu \mathrm{W}$ at $100 \mathrm{mK}$ [5]. The detectors under test are shielded by a tin-plated copper can, with light-tight feedthroughs for wiring and an absorbing labyrinth where it attaches to its base, all surrounded by a Cryoperm can (see Fig. 2). We have verified that this provides good magnetic shielding and is lighttight [6]. The detector block is mounted on a table with a thermometer and heater to allow PID control of the detector-table temperature. The temperature of the illuminator is also controlled by a PID control loop. The F-number of the optical source is 1.5 and is defined by a $12-\mathrm{mm}$-diameter aperture located $17 \mathrm{~mm}$ in front of the horn, in the 3-K shield of the black-body illuminator. The measured F\# of the horn is approximately 4.5 and the beam is therefore fully illuminated by the source. Given the band-pass of our filter stack $(33-50 \mu \mathrm{m})$, and the low temperature of the black-body illuminator ( $<32 \mathrm{~K}$ to not saturate the detectors), we initially assumed that the horn admitted only a single mode. Under this assumption the expected throughput of the system is $A \Omega=\lambda^{2}$. The power incident on the detector for this single mode and two polarizations is then (assuming a source emissivity of 1)

$$
P_{\text {inc }}(T)=\int \frac{2 h c^{2}}{\lambda^{5}} \frac{1}{e^{\frac{h c}{\lambda k T}}-1} \tau(\lambda) \lambda^{2} d \lambda
$$

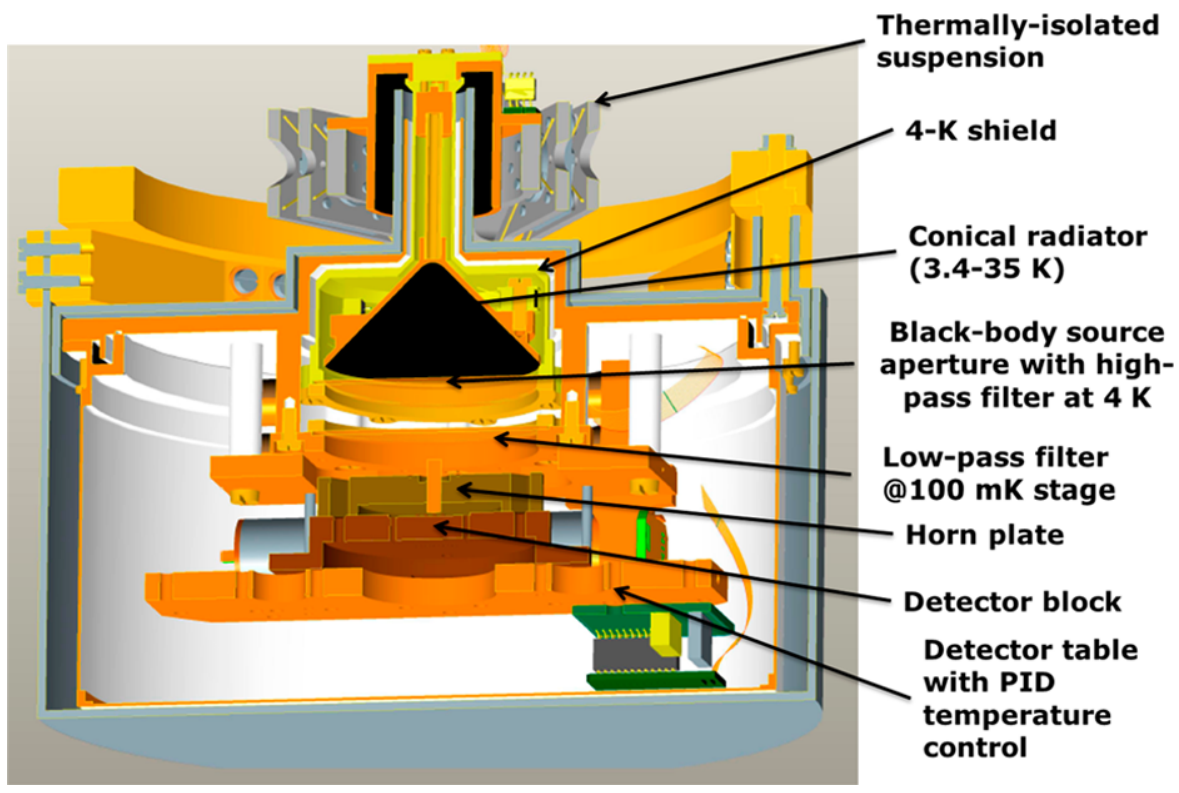

Fig. 2 Cutaway view of detector box showing the black-body illuminator and the detector table inside its nested magnetic shields. The diameter of the outer, Cryoperm shield is $15 \mathrm{~cm}$ (Color figure online) 
Fig. 3 Detected optical power vs expected optical power assuming a single mode with two polarizations propagating through the horn. Reducing the gap between the horn mouth and detector from 230 to $50 \mu \mathrm{m}$ increased the optical efficiency by a factor of 2.2 (Color figure online)

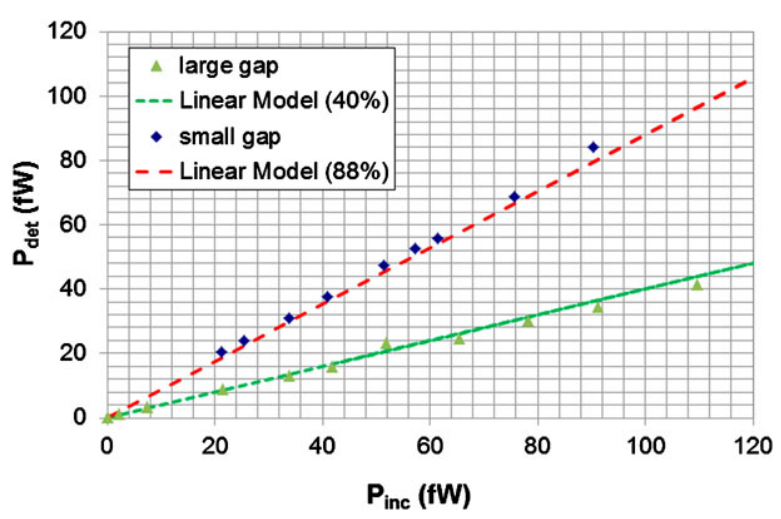

where $\tau(\lambda)$ is the measured transmission of the filter stack. The single mode optical efficiency of the horn-cavity-detector combination is then

$$
\eta=\frac{P_{\mathrm{det}}(T)}{P_{\mathrm{inc}}(T)}
$$

where $P_{\text {det }}(T)$ is the detected optical power at illuminator temperature $T$.

\section{Measurements}

For optical measurements we regulated the detector-table temperature at a fixed value (usually $70 \mathrm{mK}$ ) and recorded IV curves at different illuminator temperatures from which we derived the absorbed optical power. We also measured the noise in the detectors at different illuminator temperatures and found that the measured photon noise agrees with the value expected from the predicted incident power. We originally derived an optical efficiency of $40 \%$ relative to a single mode propagating in the horn when the distance between the TES and the horn mouth was $230 \mu \mathrm{m}$ [7]. We expected that a smaller distance between the feedhorn and detector would give a higher coupling efficiency so we modified the horn plate to reduce this distance to $50 \mu \mathrm{m}$. As shown in Fig. 3, the detected optical power increases by a factor of 2.2 when the gap is reduced. The single-mode efficiency is then $88 \%$. Again, the measured optical NEP is consistent with this higher optical efficiency. It should be noted that these efficiencies assume a single mode propagating in the horn. We expect this assumption to underestimate the optical efficiency because of the presence of higher-order modes.

\section{Second-Generation Optical Calibrator}

We successfully measured the optical response of SAFARI prototype detectors all the way to saturation using the illuminator shown in Fig. 2. However, because of the low temperature of the black-body emitter (3.4-34 K), the pass-band of the short-wave 


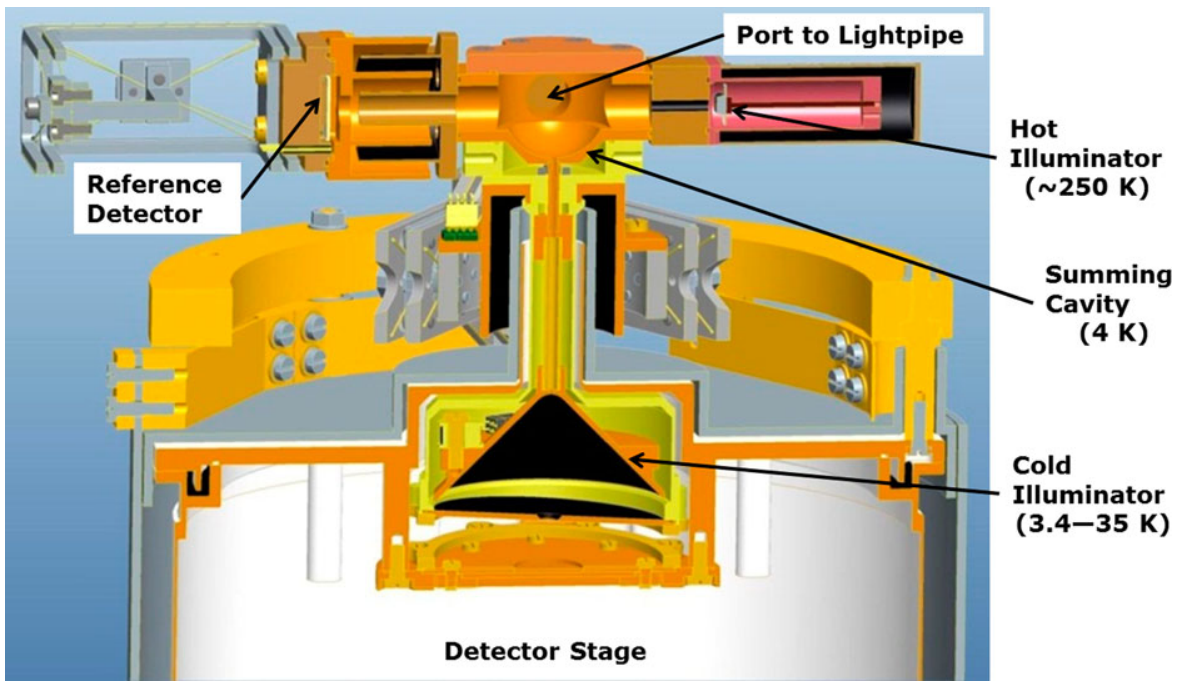

Fig. 4 Second-generation optical illuminator with hot and cold black-body illuminators, reference detectors and port for lightpipe to outside (Color figure online)

detectors is illuminated by the Wien tail of the black-body spectrum. This means that only the long-wavelength end of the pass-band sees significant power. In order to illuminate the pass-band more uniformly with a black-body source, we need that source to have a much higher temperature. We have thus added a hot (200-300 K) source, with cold attenuation to reduce the power, as shown in Fig. 4. In addition, there is a light-pipe to room temperature so that we can inject power from an external FTS or a modulated source. The light-pipe has a two-vane cold shutter that can also be chopped at $\sim 20 \mathrm{~Hz}$ for lock-in measurements. A reference detector allows us to measure the spectral content of the radiation. The reference detector is a TES bolometer, similar to that shown in Fig. 1, suspended in a large absorbing cavity so that its optical absorption coefficient is flat over a broad wavelength range. To increase the dynamic range of this reference detector we bias the TES in series with the Ta absorber, giving a double-transition TES which greatly increases the dynamic range without affecting the sensitivity for low optical loads. The light-pipe, reference detector, and hot illuminator are attached to a reflective summing cavity that has a temperature of $4 \mathrm{~K}$. A second light pipe, with diameter $4.5 \mathrm{~mm}$, leading to a hole in the center of the cold illuminator allows radiation from the summing cavity to illuminate the detectors under test. Both the hot and cold illuminators can be operated during the same cool-down, allowing cross-calibration. This second-generation optical calibrator is now being used for detector measurements in the SAFARI Detector Test Facility. We have measured interferograms from an external Michelson interferometer and verified that the reference detector has a flat spectral response from 1 to at least $7 \mathrm{THz}$. 


\section{Discussion}

With the large $(230-\mu \mathrm{m})$ gap between horn and detector and the horn fully illuminated we obtained virtually identical efficiencies, $\eta=40 \%$ assuming a single mode, for two similar detectors, even though the detectors have slightly different thermal properties. We note that this $40 \%$ efficiency was also consistent with the measured photon noise subject to the same assumptions about throughput. When the gap between the detector and horn is reduced to $50 \mu \mathrm{m}$ we see an increase in optical efficiency by a factor of 2.2. We estimate that with the large illuminator aperture the single-mode approximation can overestimate the optical efficiency by a factor of up to 2.45 because two higher-order circular-waveguide modes are admitted as well as the fundamental $\mathrm{TE}_{11}$ mode: $\mathrm{TE}_{21}$ (two polarizations) and $\mathrm{TM}_{01}$ (one polarization). This gives optical efficiencies of 16 and $36 \%$ for gaps of 230 and $50 \mu \mathrm{m}$, respectively compared with $31 \%$ and $66 \%$ predicted by electromagnetic simulations. This is not unreasonable since the simulations assumed that the band was illuminated uniformly whereas the low-temperature black-body illuminator that we have used illuminates mostly the long-wavelength end of the band. To resolve the ambiguity of the filledbeam measurements we are carrying out measurements with small apertures that will eliminate the off-axis contribution of the higher-order modes.

\section{Conclusions}

We have measured optical efficiency in good agreement with models for TES bolometers designed for SAFARI's short-wavelength band $(33-60 \mu \mathrm{m})$ in combination with a spherical backshort and conical horn.

Acknowledgements We thank Douglas Griffin of the Rutherford Appleton Laboratory for supplying the feedhorn. The detectors and feedhorn used in this work were originally produced for ESA TRP contract No. 22359/09/NL.CP, TES Spectrometer.

\section{References}

1. B. Swinyard et al., Exp. Astron. 23, 193 (2008)

2. B. Jackson et al., IEEE Trans. Terahertz Sci. Technol. 2, 1 (2011)

3. K.D. Irwin, G.C. Hilton, in Cryogenic Particle Detection, ed. by C. Enss (Springer, Berlin, 2005), pp. $81-97$

4. P. Khosropanah et al., Proc. SPIE 8452, 845209 (2012)

5. Leiden Cryogenics BV. http://www.leidencryogenics.com

6. M.D. Audley et al., J. Low Temp. Phys. 167, 208 (2012)

7. M.D. Audley et al., Proc. SPIE 8452, 84520B (2012) 